\title{
Learned and unlearned components of the rat's adaptation to water deprivation ${ }^{1}$
}

\author{
GLENN I. HATTON AND C. ROBERT ALMLI ${ }^{2}$ \\ MICHIGAN STATE UNIVERSITY
}

Rats were given three successive 10-day adaptation periods on $23.5 \mathrm{~h}$ water deprivation with five days of ad lib conditions interpolated between adaptations. Latency to drink, water intake, and body weights were measured. Latency to drink decreased with repeated testing, indicating a learned component of adaptation behavior. Water intake and body weight, conversely, required the same adjustment process during each adaptation, indicating that these components are not influenced by prior learning. The results are discussed in the context of a prior controversy in the literature.

A controversy has resulted from the behavioral studies which have attempted to describe the process of adaptation to water deprivation. Ghent (1957) reported decreasing latency to drink, increasing water intake, and progressively less weight loss during successive days of adaptation to $23 \mathrm{~h}$ water deprivation. She explained the increased intake on successive days as a learning phenomenon. She reasoned that since there was progressivley less weight loss, the increased intake could not be due to an increased physiological deficit. However, she did not test this explanation with repeated adaptations. Beck (1962) found that rats deprived of water one day and allowed two days on ad lib before being deprived again, showed no progressive increase in intake with repeated deprivations. Beck reasoned that either the rat did not learn to utilize the drinking period in spite of repeated deprivations, or the need for water was less on the first day of deprivation. He concluded that increased water intake with experience on deprivation was due to increased need for water.

The present experiment attempted to resolve this controversy by using (a) 10 day adaptation periods within which drinking is known to stabilize, and (b) constant external stimulus conditions during the ad lib recovery periods, which eliminates confounding factors associated with a novel drinking situation. Method

The Ss were 11 male albino rats of the Holtzman strain. They were housed in individual wire cages, where Wayne Lab Blox were constantly available. Three days after their arrival in the laboratory, Ss were weighed and the water bottles were removed from their home cages. For the next 10 days, Ss were weighed and given $0.5 \mathrm{~h}$ free access to water in individual drinking boxes. These boxes were fixed with $100 \mathrm{ml}$ gas measuring tubes, graduated in $0.2 \mathrm{ml}$, and had Plexiglas covers for observations of drinking. Latency to drink after being placed in the box and water intake were measured daily. Following the 10th day on this schedule, ad lib conditions were begun and Ss were randomly divided into two groups. Six Ss were placed in the drinking boxes for $0.5 \mathrm{~h}$ at their usual daily drinking time, and the remaining five Ss were placed in individual compartments in a carrying cage. This continued for five days, during which all Ss were weighed as before and intakes were recorded for those Ss which were in the drinking boxes. A second 10-day adaptation period, identical to the first followed the five days of ad lib conditions, after which Ss were again placed on ad lib. This second ad lib period was like the first, except that all Ss were placed in the drinking boxes for $0.5 \mathrm{~h}$ at their usual daily drinking time. A third 10-day adaptation period was then given, and was identical to the first two described above.

Results

The results are summarized in Fig. 1. The top panel of the figure presents the means for reciprocal latency to drink as a function of days of the experiment. Analyses of these data showed the following: (a) There was no overlap of the distributions of re-

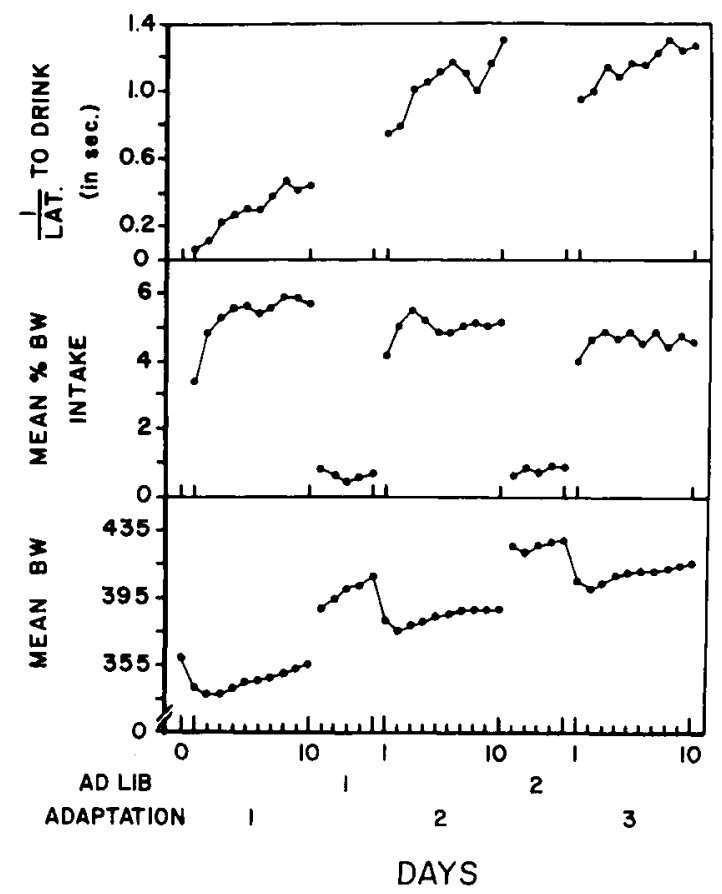

Fig. 1. Mean reciprocal hatency to drink, mean percent body weight intake, and mean body weight plotted as a function of days of the experiment. Ad lib and adaptation periods are as indicated below the baseline. 
ciprocal latencies for Day 1 and Day 10 of Adaptation 1. (b) There was no reliable difference between the "carrying cage" Ss and the "drinking box" Ss in latency to drink on the first day of Adaptation 2, although there was an overall significant difference between Day 10 of Adaptation 1 and Day 1 of Adaptation $2(t=2.08, d f=10, p<.05)$ when the two groups were combined. (c) The latencies continue to shorten during Adaptation 2, but on Day 1 of Adaptation 3, mean reciprocal latency was reliably lower than it was on the last day of the previous adaptation period $(t=$ 2.46, df $=10 . p<.025$ ).

The center panel of the figure summarizes the water intake, expressed as a percentage of body weight. The differences between the Day 1 and the Day 10 means for each adaptation period were all significant at $p$ $<.005$. The mean intake on the first day of Adaptation 1 was reliably below the means for the corresponding day of the second and third adaptation period $(p<$ .025).

Plotted in the bottom panel of the figure are the mean body weights for the duration of the experiment. The body weight loss from the last day of ad lib conditions to the second day of each corresponding adaptation period was significant at $\mathrm{p}<.001$. These weight losses were roughly equivalent in all three cases, the largest being associated with Adaptation 2.

\section{Discussion}

The latency data definitely show that some familiarization with the drinking situation was important in obtaining rapid orientation to the water spout. Further, the increased speed of starting to drink on Day 1 of Adaptation 2 indicates that the initial familiarization produced behavioral changes which not only persisted, but became even more pronounced during the ad lib recovery period. By the last day of the second adaptation period, reciprocal latency appeared to reach a maximum value. On Adaptation 3, unlike Adaptation 2, there was significant decrease in this measure, with a subsequent gradual return to the previously established maximum. Thus, rapid approach to the water spout could be interpreted as analogous to or as actually being a motor skill, which takes some time to develop to a high level. Decrements, such as the one at the beginning of Adaptation 3 , are to be expected if practice is interrupted as it was in this case. This interpretation is fairly consistent with that of Ghent (1957). Explanations of the decrement at the beginning of Adaptation 3, which are based on some altered re- inforcement value of the drinking boxes during the ad lib recovery period, run Into the difficulty presented by the increment shown in Adaptation 2.

The percent body weight intake data are consistent with the findings of Beck (1962). The Ss do not seem to learn to increase their Day 1 intakes with seccessive adaptations, as evidenced by the fact that the means for Day 1 of Adaptations 2 and 3 are not different. The lower intake associated with the first day of Adaptation 1 was to be anticipated on the basis of the novelty of the drinking situation. This result is consistent with the findings of Fink \& Patton (1953). Drinking on the first day of Adaptation 2 was slightly but significantly higher for the "carrying cage" Ss than it was for the "drinking box" Ss. It seems that this result can be accounted for by the following considerations. Neither group found water in the home cage upon returning there on the last day of ad lib conditions. Thus, Ss retained in the carrying cage experienced $0.5 \mathrm{~h}$ more of water deprivation, during which time all Ss of the other group drank from 1-5 ml of water.

It seems that Beck's reasoning from increased water need is correct on the basis that, as adaptation to deprivation proceeds, rats gain weight. This gain is in large part due to the fact that they eat more food. This they do by slowly adjusting their eating times, so that the major part of their food intake immediately follows the daily drinking time. The osmotic stress caused by the increased food intake results in higher water intake on the following day, which in turn results in higher food intake, etc. Apparently, this process continues with diminishing overshoots until asymptotic intake and weights are reached. Many regulatory control systems show gradual damping effects of this sort (Bayliss, 1966).

\section{References}

BAYLISS, L. E. Living control systems. San Francisco: W. H. Freeman and Co., 1966.

BECK, R. C. The rat's adaptation to a 23.5 -hour water-deprivation schedule. J. comp. physiol. Psychol., 1962, 55, 646-648.

FINK, J. B., \& PATTON, R. M. Decrement of a learned drinking response accompanying changes in several stimulus characteristics. $J$. comp. physiol. Psychol, 1953, 46, 23-27.

GHENT, L. Some effects of deprivation on eating and drinking behavior. J. comp. physiol. Psychol., 1957, 50, 172-176.

Notes

1. This research was supported, in part, by grant no. MH 14270 from NIMH.

2. NIH Trainee on Training grant no. MH 10611. 